\title{
Observer-Based Adaptive Fuzzy Predefined Performance Control of a Class of Nonlinear Pure-Feedback Systems with Input Delay
}

\author{
Xin Li, ${ }^{1}$ Qiang Zhang, ${ }^{1}$ and Dakuo He $\mathbb{D}^{1,2}$ \\ ${ }^{1}$ College of Information Science and Engineering, Northeastern University, Shenyang 110819, China \\ ${ }^{2}$ State Key Laboratory of Synthetical Automation for Process Industries and the College of Information Science and Engineering, \\ Northeastern University, Shenyang 110819, China
}

Correspondence should be addressed to Dakuo He; hedakuo@ise.neu.edu.cn

Received 17 September 2020; Revised 19 October 2020; Accepted 30 October 2020; Published 10 December 2020

Academic Editor: Heng Liu

Copyright (C) 2020 Xin Li et al. This is an open access article distributed under the Creative Commons Attribution License, which permits unrestricted use, distribution, and reproduction in any medium, provided the original work is properly cited.

This paper presents a problem of observer-based adaptive fuzzy predefined performance control of a class of nonlinear purefeedback systems with input delay and unknown control direction. Compared with the existing research, a novel predefined performance controller is proposed, which relaxes the assumption that the initial error is known. In addition, it is difficult to design the controllers due to input delay and nonaffine properties of the pure-feedback systems, which can be simplified by Pade approximation. Moreover, dynamic surface control and Nussbaum functions are applied to overcome the calculation explosion caused by repeated differentiations and unknown control direction, respectively. Based on the above methods, an adaptive fuzzy predefined performance controller is proposed, and it is proved that all the signals of a closed-loop system are semiglobally uniformly ultimately bounded (SGUUB). The tracking errors converge within a predefined range, while the observer estimation errors converge within a small zero region. Finally, the simulation results demonstrate the effectiveness of the proposed control system.

\section{Introduction}

In the past years, the adaptive nonlinear systems based on the backstepping method has matured increasingly and received widespread attention in $[1,2]$. At an earlier time, there was an unmodeled nonlinear problem in the above system, which greatly limited the application of this technology. To solve the above problems, fuzzy logic systems (FLSs) and neural networks (NNs) were applied extensively to approximate unknown nonlinear function in [3-7]. However, the characteristic of the backstepping method is a class of recursive design procedures coupled with Lyapunov function candidates; hence, the repeated differentiation of virtual controller leads to the complexity explosion problem. Afterwards, the dynamic surface control (DSC) technology was integrated into the backstepping method to solve this problem in [8-10]. In addition, since the unmeasurable state in the application has a great restriction, the state observer was employed to estimate the unmeasured state in [11-15]. Among them, an equivalent output injection sliding mode observer was proposed in [12], which could estimate the status of each follower and its neighbor. And high gain observer was used to estimate the position, course, and speed of the vessel in [13]. In recent years, observer-based adaptive fuzzy control with the DSC technology was investigated in [16-18].

It is well known that different from strict-feedback systems, pure-feedback systems have nonaffine structure of the variables, which presents more challenges to the controller design. Fortunately, the mean value theorem was proposed to solve the variables coupling problem of nonaffine structures in $[19,20]$. Moreover, pure-feedback systems usually have the problem of unknown input control direction, which could be solved by Nussbaum functions in [21-24]. In addition, the input and output of the control systems have many restrictions, such as input saturation, dead zone, and input delay in [25-33]. It is worth 
mentioning that an adaptive predictor incorporated with a high-order neural network observer was proposed to obtain the predictions of the future system states in pure-feedback systems in [28], which were applied in the control design to avoid the input delay and nonlinearities. Subsequently, the input delay was solved by Pade approximation technique and intermediate variables in the strict-feedback systems in [29-31], which simplified the controller design. However, to the best of the author's knowledge, the combination of input delay and pure-feedback systems was rarely considered. Therefore, the controller design of pure-feedback systems with input delay is complicated, which needs to be further developed.

On the other side, the predefined control performance is better able to achieve the desired performance, such as overshoot, convergence rate, and convergence accuracy. Therefore, the prescribed performance control was proposed, which can satisfy preset transient and steady-state tracking performance in [34-36]. In particular, an adjustable finite-time prescribed performance function with fast convergence speed was adopted in $[37,38]$, which ensures realtime adjustment of controller parameters cased by the tracking error. Although the research of the prescribed performance control method is approaching maturity, there was still limitation of unknown initial values. Fortunately, a predefined performance function with time-varying design parameters was proposed to reduce the impact of unknown initial tracking error in [39]. However, it is not applied to the unknown nonlinear pure-feedback systems. In summary, the existing predefined performance control methods are insufficient to deal with a class of nonlinear pure-feedback systems with input delay. Therefore, the controller design for the above conditions needs to be developed.

Based on the above discussion, this article presents a method for observer-based adaptive fuzzy predefined performance control of a class of nonlinear pure-feedback systems with unknown control direction and input delay. State observer and FLSs are proposed to solve the problem of approximate unmeasurable state and unknown nonlinear functions, respectively. Compared with the existing literature, the main contributions of this paper are as follows:

(1) In the existing literatures [34, 37], the initial values in predefined performance control are assumed to be known. In order to relax that assumption, a novel predefined performance control method is proposed, which is a variable-parameter scheme independent of the initial error. Therefore, the restriction of the unknown initial error in the predefined performance control is solved.

(2) Compared with [36], the input delay is introduced into the pure-feedback systems. It is difficult to design the controllers due to input delay and nonaffine properties of the pure-feedback systems, which can be simplified by Pade approximation and mean value theorem, respectively.

(3) By combining DSC technology and backstepping method, the issue of complexity explosion caused by repeated differentiations of some intermediate variables is eliminated. And the Nussbaum functions are proposed to solve unknown control direction.

The framework of this article is as follows. In Section 2, preliminaries and problem formulation are presented. In Section 3, an observer-based adaptive fuzzy predefined performance controller is designed of a class of nonlinear pure-feedback systems with unknown control direction and input delay, and the stability analysis is given. In Section 4, an example simulation is given to verify the feasibility of the proposed method. Finally, the Section 5 is the conclusions and the prospect of the future work.

Notations: $\mathfrak{R}$ denotes the set of real numbers, $\mathfrak{R}^{i}$ denotes the $i$-dimensional vector space, and $R_{+}$is the set of all nonnegative real numbers. $\|\cdot\|$ indicates the Euclidean norm of vectors or matrix. For a matrix $X, X^{T}$ indicates its transpose and $X^{-1}$ indicates its inverse. For a matrix $Q$, $\lambda_{\min }(Q)$ stands for the smallest eigenvalue of $Q$ and $\lambda_{\max }(Q)$ stands for the largest eigenvalue of $Q$.

\section{Preliminaries and Problem Formulation}

2.1. System Descriptions and Assumptions. A class of nonlinear pure-feedback systems with input delay is considered as

$$
\left\{\begin{array}{l}
\dot{x}_{i}=f_{i}\left(\underline{x}_{i}, x_{i+1}\right)+d_{i}(t), \quad 1 \leq i \leq n-1, \\
\dot{x}_{n}=f_{n}\left(\underline{x}_{n}, u(t-\delta)\right)+d_{n}(t), \\
y=x_{1}
\end{array}\right.
$$

where $\underline{x}_{i}=\left[x_{1}, \ldots, x_{i}\right]^{T} \in \mathfrak{R}^{i}$ are the state vector, $y \in \mathfrak{R}$ is the output, $f_{i}\left(\underline{x}_{i}, x_{i+1}\right), f_{n}\left(\underline{x}_{n}, u(t-\delta)\right)$ are unknown smooth functions, $d_{i}(t)$ means unknown and bounded external disturbance inputs, and $\delta$ denotes the input delay, which is an small unknown positive constant caused by network delay. Moreover, the output $y$ is measurable.

Because of the coupling between states $x_{i+1}$ and $u(t-\delta)$ in smooth functions $f_{i}\left(\underline{x}_{i}, x_{i+1}\right)$ and $f_{n}\left(\underline{x}_{n}, u(t-\delta)\right)$, which makes the desired control objectives difficult to design, the mean value theorem is used as

$$
\begin{aligned}
f_{i}\left(\underline{x}_{i}, x_{i+1}\right) & =f_{i}\left(\underline{x}_{i}, 0\right)+g_{i}\left(\underline{x}_{i}, x_{i+1}\right) x_{i+1}, \\
f_{n}\left(\underline{x}_{n}, u(t-\delta)\right) & =f_{n}\left(\underline{x}_{n}, 0\right)+g_{n}\left(\underline{x}_{n}, u(t-\delta)\right) u(t-\delta),
\end{aligned}
$$

where $g_{i}\left(\underline{x}_{i}, x_{i+1}\right)=\partial f_{i}\left(\underline{x}_{i}, x_{i+1}\right) /\left.\partial x_{i+1}\right|_{x_{i+1}^{0}}$ and $g_{n}\left(\underline{x}_{n}\right.$, $u(t-\delta))=\partial f_{n}\left(\underline{x}_{n}, u(t-\delta)\right) /\left.\partial u(t-\delta)\right|_{u_{(t-\delta)}^{0}}, x_{i+1}^{0}$ is certain point between zero and $x_{i+1}$, and $u_{(t-\delta)}^{0}$ is certain point between zero and $u(t-\delta)$. Let $f_{i}\left(\underline{x}_{i}, 0\right)=f_{i}\left(\underline{x}_{i}\right)$, $f_{n}\left(\underline{x}_{n}, 0\right)=f_{n}\left(\underline{x}_{n}\right), \quad g_{i}\left(\underline{x}_{i}, x_{i+1}\right)=g_{i}, \quad$ and $\quad g_{n}\left(\underline{x}_{n}, u\right.$ $(t-\delta))=g_{n}$.

Substituting (2) into system (1), one can obtain as

$$
\left\{\begin{array}{l}
\dot{x}_{i}=f_{i}\left(\underline{x}_{i}\right)+g_{i} x_{i+1}+d_{i}(t), \\
\dot{x}_{n}=f_{n}\left(\underline{x}_{n}\right)+g_{n} u(t-\delta)+d_{n}(t), \\
y=x_{1} .
\end{array}\right.
$$


To get the actual control input $u$ by removing the effect of input delay $\delta$, the Pade approximation method and the delay theorem of Laplace transform can be used, which solve the analysis complexity problem caused by time delay, and it follows that

$$
\ell\{u(t-\delta)\}=e^{-\delta s} \ell(u(t)) \approx \frac{1-\delta s / 2}{1+\delta s / 2} \ell\{u(t)\},
$$

where $s$ represents the Laplace variable and $\ell\{u(t)\}$ is the Laplace transform of $u(t)$.

Define the intermediate variable $x_{n+1}$ as

$$
\frac{1-\delta s / 2}{1+\delta s / 2} \ell\{u(t)\}=\ell\left\{x_{n+1}\right\}-\ell\{u(t)\} .
$$

By transforming formula (5), one can be given as

$$
2 \ell\{u(t)\}=\ell\left\{x_{n+1}(t)\right\}+\frac{\delta s}{2} \ell\{u(t)\} .
$$

By taking the inverse Laplace transform,

$$
\dot{x}_{n+1}=2 \beta u-\beta x_{n+1} \text {, }
$$

where $\beta=2 / \delta$ is a variable.

Remark 1. Pade approximation has been used in [31]. In this article, since the Pade approximation is applied to solve a class of small time delay problems, $e^{-\delta s}$ is approximately equal to $1-\delta s / 2 /(1+\delta s / 2)$ when the time delay is very small. And the intermediate variable $x_{n+1}$ is not a real variable of system (1), which can be viewed as an error variable. And this has been verified in the simulation in [31].

By using the above methods, (3) can be further written as

$$
\left\{\begin{array}{l}
\dot{x}_{i}=f_{i}\left(\underline{x}_{i}\right)+g_{i} x_{i+1}+d_{i}(t) \\
\dot{x}_{n}=f_{n}\left(\underline{x}_{n}\right)+g_{n} x_{n+1}-g_{n} u+d_{n}(t), \\
\dot{x}_{n+1}=-\beta x_{n+1}+2 \beta u \\
y=x_{1}
\end{array}\right.
$$

Assumption 1 (see [9]). The expected signal $y_{d}$ and its derivatives $\dot{y}_{d}$ and $\ddot{y}_{d}$ are all known and bounded, which is $\Upsilon=\left\{y_{d}, \dot{y}_{d}, \ddot{y}_{d}: y_{d}^{2}+\dot{y}_{d}^{2}+\ddot{y}_{d}^{2} \leq \bar{Y}\right\}$, where $\bar{Y}$ is a positive constant.

Assumption 2 (see [20]). The sign of $g_{i}$ is unknown, but $g_{i}$ has the same sign and its public super bound is known, which is $0<\left|g_{i}\right|<g^{*}$.

Assumption 3. The disturbance $d_{i}$ is bounded to a positive constant $d_{i}^{*}$, that is, $\left|d_{i}\right| \leq d_{i}^{*}$.

Assumption 4 (see [27]). There is a known constant $s_{i}$ that satisfies $f_{i}\left(\underline{x}_{i}\right)-f_{i}\left(\underline{\hat{x}}_{i}\right) \leq s_{i}\left\|\underline{x}_{i}-\underline{\hat{x}}_{i}\right\|$, where $\hat{x}_{i}=\left[\hat{x}_{1}, \hat{x}_{2}, \ldots\right.$, $\left.\hat{x}_{i}\right]^{T}$ is the estimate of $\underline{x}_{i}=\left[x_{1}, x_{2}, \ldots, x_{i}\right]^{T}$, and $\|X\|$ represents the 2 norm of the vector $X$.
2.2. Fuzzy Logic Systems. Because the nonlinear function is unknown, FLSs is proposed. Build FLSs with the if-then rules.

$R^{q}:$ if $x_{1}$ is $F_{1}^{q}$ and $x_{2}$ is $F_{2}^{q}$ and ... and $x_{n}$ is $F_{n}^{q}$. Then, $y$ is $B^{q}, q=1,2, \ldots, a$. Here, $x=\left[x_{1}, \ldots, x_{n}\right]^{T}$ and $y$ are the FLS input and output, respectively. Fuzzy sets $F_{i}^{q}$ and $B^{q}$, associated with the fuzzy functions $\mu_{F_{i}^{q}}\left(x_{i}\right)$ and $\mu_{B^{q}}(y)$, respectively. $a$ is the rules number. Thus, FLS can be calculated by formula

$$
y(x(t))=\frac{\sum_{q=1}^{a} \tilde{y}_{q} \prod_{i=1}^{n} \mu_{F_{i}^{q}}\left(x_{i}\right)}{\sum_{q=1}^{a}\left(\prod_{i=1}^{n} \mu_{F_{i}^{q}}\left(x_{i}\right)\right)},
$$

where $\widetilde{y}_{q}=\max _{y \in R} \mu_{B^{q}}(y)$.

Let $\varphi_{q}=\prod_{i=1}^{n} \mu_{F_{i}^{q}}\left(x_{i}\right) / \sum_{q=1}^{a}\left(\prod_{i=1}^{n} \mu_{F_{i}^{q}}\left(x_{i}\right)\right)$ and denote $\theta=\left[\widetilde{y}_{1}, \widetilde{y}_{2}, \ldots, \widetilde{y}_{a}\right]^{T^{i}}=\left[\theta_{1}, \theta_{2}, \ldots, \theta_{a}\right]^{T}$ and $\varphi^{T}(x)=$ $\left[\varphi_{1}(x), \ldots, \varphi_{a}(x)\right]$; then, FLS can be rewritten as $y(x)=\theta^{T} \varphi(x)$.

Lemma 1 (see [40]). Let $f(x)$ be a continuous function defined on a compact set $\Omega$. Then, for any constant $\varepsilon>0$, there exists an FLS such as

$$
\sup _{x \in \Omega}\left|f(x)-\theta^{T} \varphi(x)\right| \leq \varepsilon .
$$

Define the idealized parameter vector $\theta_{i}^{*}$ as

(i) $\theta_{1}^{*}=\arg \min _{\theta_{1} \in \Omega_{1}}\left[\sup _{x_{1} \in U_{1}}\left|\hat{f}_{1}\left(x_{1} \mid t \theta_{1}\right)-f_{1}\left(x_{1}\right)\right|\right]$

(ii) $\theta_{i}^{*}=\operatorname{argmin}_{\theta_{i} \in \Omega_{i}}\left[\sup _{\hat{x}_{i} \in U_{i}}\left|\widehat{f}_{i}\left(\underline{\hat{x}}_{i} \mid t \theta_{i}\right)-f_{i}\left(\underline{\hat{x}}_{i}\right)\right|\right], \quad(i=$ $2, \ldots, n)$

Here, $\Omega_{1}, \Omega_{i}, U_{1}$, and $U_{i}$ are compact for $\theta_{1}, \theta_{i}, x_{1}$, and $\underline{\hat{x}}_{i}$, respectively.

By Lemma 1, the nonlinear functions can be approximated by the following FLSs:

$$
\begin{aligned}
\widehat{f}_{1}\left(x_{1} \mid \theta_{1}\right) & =\theta_{1}^{T} \varphi_{1}\left(x_{1}\right), \\
\hat{f}_{i}\left(\underline{\hat{x}}_{i} \mid \widehat{\theta}_{i}\right) & =\widehat{\theta}_{i}^{T} \varphi_{i}\left(\underline{\hat{x}}_{i}\right), \quad i=2, \ldots, n .
\end{aligned}
$$

The fuzzy minimum approximation errors can be defined as $\varepsilon_{1}\left(x_{1}\right)=f_{1}\left(x_{1}\right)-\widehat{f}_{1}\left(x_{1} \mid \theta_{1}^{*}\right)$ and $\varepsilon_{i}\left(\widehat{x}_{i}\right)=f_{i}$ $\left(\hat{x}_{i}\right)-\widehat{f}_{i}\left(\hat{x}_{i} \mid \theta_{i}^{*}\right)$, where $\underline{x}_{i}$ are the estimation of the state $\underline{x}_{i}$.

Assumption 5. The approximation error $\varepsilon_{i}$ is bounded, and there is a constant $\varepsilon_{i}^{*}$ that satisfies $\left|\varepsilon_{i}\right| \leq \varepsilon_{i}^{*}$.

From (11), system (8) can be expressed as

$$
\left\{\begin{array}{l}
\dot{x}_{i}=g_{i} x_{i+1}+\theta_{i}^{* T} \varphi_{i}\left(\underline{\hat{x}}_{i}\right)+\varepsilon_{i}\left(\underline{\hat{x}}_{i}\right)+d_{i}+\Delta f_{i} \\
\dot{x}_{n}=g_{n} x_{n+1}-g_{n} u+\theta_{n}^{* T} \varphi_{n}\left(\underline{\hat{x}}_{n}\right)+\varepsilon_{n}\left(\underline{\hat{x}}_{n}\right)+d_{n}+\Delta f_{n} \\
\dot{x}_{n+1}=-\beta x_{n+1}+2 \beta u \\
y=x_{1}
\end{array}\right.
$$

where $\Delta f_{i}=f_{i}\left(\underline{x}_{i}\right)-f_{i}\left(\underline{\hat{x}}_{i}\right), i=2, \ldots, n$.

Rewriting (12) in the following formula, 


$$
\left\{\begin{array}{l}
\dot{x}=A x+\bar{F}^{*}+\varepsilon+d+\Delta f+E_{n} u, \\
y=E_{0}^{T} x,
\end{array}\right.
$$

where

$$
x=\left[x_{1}, x_{2}, \ldots, x_{n}, x_{n+1}\right]^{T},
$$

$\left[\begin{array}{cccccc}0 & g_{1} & 0 & \ldots & 0 & 0 \\ 0 & 0 & g_{2} & \ldots & 0 & 0 \\ \vdots & & & & & \\ 0 & 0 & 0 & \ldots & g_{n-1} & 0 \\ 0 & 0 & 0 & \ldots & 0 & g_{n} \\ 0 & 0 & 0 & \ldots & 0 & -\beta\end{array}\right], \quad \varepsilon=\left[\varepsilon_{1}\left(x_{1}\right), \varepsilon_{2}\left(\underline{x}_{2}\right), \ldots, \varepsilon_{n}\left(\underline{x}_{n}\right)\right.$,

$0]^{T}, \quad \bar{F}^{*}=\left[\theta_{1}^{* T} \varphi_{1}\left(x_{1}\right), \theta_{2}^{* T} \varphi_{2}\left(\underline{\underline{x}}_{2}\right), \ldots, \theta_{n}^{* T} \varphi_{n}\left(\underline{\hat{x}}_{n}\right), 0\right]^{T}$, $d=\left[d_{1}, d_{2}, \ldots, d_{n}, 0\right]^{T}, \quad \Delta f=\left[0, \Delta f_{2}, \ldots, \Delta f_{n}, 0\right]^{T}, \quad E_{0}^{T}=$ $[1,0, \ldots, 0]$, and $E_{n}^{T}=\left[0, \ldots, 0,-g_{n}, 2 \beta\right]$.

2.3. Nussbaum-Type Function. A continuous function $N(\mu)$ is called the Nussbaum function if it has the following properties:

$$
\begin{aligned}
& \lim _{m \longrightarrow \infty} \sup \frac{1}{m} \int_{0}^{m} N(\mu) \mathrm{d} \mu=\infty, \\
& \lim _{m \longrightarrow \infty} \inf \frac{1}{m} \int_{0}^{m} N(\mu) \mathrm{d} \mu=-\infty,
\end{aligned}
$$

where $m$ is the integral upper boundary. For instance, the frequently used continuous Nussbaum-type functions contain $\mu^{2} \cos (\mu), \mu^{2} \sin (\mu), e^{\mu^{2}} \cos (\mu)$, and so on. In this work, the continuous Nussbaum-type function $N(\mu)=\mu^{2} \cos (\mu)$ is utilized.

Lemma 2 (see $[41,42]$ ). Smooth functions $V(\cdot)$ and $\mu(\cdot)$ are defined on $\left[0, t_{f}\right)$, where $V(t) \geq 0\left(\forall \in\left[0, t_{f}\right)\right)$ and $N(\mu)$ is a Nussbaum-type function. If the following inequality holds

$$
V(t) \leq c \pm \int_{0}^{t} \sum_{l=1}^{n}\left(g N\left(\mu_{l}\right)+1\right) \dot{\mu}_{l} \mathrm{~d} \tau,
$$

where $g$ is a nonzero constant and $c$ represents appropriate constant, then $V(t), \mu(t)$, and $\int_{0}^{t} \sum_{l=1}^{n}\left(g N\left(\mu_{l}\right)+1\right) \dot{\mu}_{l} d \tau$ must be bounded on $\left[0, t_{f}\right)$.

Remark 2. The parameters $g_{i}$ and $g_{n}$ are time-varying parameters and their signs are unknown. If the control direction changes rapidly, it is difficult to effectively guarantee the stability of the closed-loop system under the selfadaptive condition. Therefore, compared with the control direction which is assumed to be known, Assumption2 is more flexible in the application. In addition, similar to [20], this paper uses the Nussbaum functions to solve the control direction problem, which relaxes the prior knowledge.

2.4. Fuzzy State Observer Design. To estimate the unmeasurable states of the system, the corresponding fuzzy observer is designed as

$$
\left\{\begin{array}{l}
\dot{\hat{x}}_{i}=g_{i} \hat{x}_{i+1}+\theta_{i}^{T} \varphi_{i}\left(\underline{\hat{x}}_{i}\right)+k_{i}\left(y-\hat{x}_{1}\right), \\
\dot{\hat{x}}_{n}=g_{n} \widehat{x}_{n+1}-g_{n} u+\theta_{n}^{T} \varphi_{n}\left(\underline{\hat{x}}_{n}\right)+k_{n}\left(y-\hat{x}_{1}\right), \\
\dot{\hat{x}}_{n+1}=-\beta \widehat{x}_{n+1}+2 \beta u, \\
\hat{y}=\hat{x}_{1} .
\end{array}\right.
$$

Rewriting (16) in the following formula,

$$
\left\{\begin{array}{l}
\dot{\hat{x}}=A_{0} \widehat{x}+K y+\bar{F}+E_{n} u, \\
\hat{y}=E_{0}^{T} \widehat{x},
\end{array}\right.
$$

where $\quad \hat{x}=\left[\widehat{x}_{1}, \hat{x}_{2}, \ldots, \hat{x}_{n}, \widehat{x}_{n+1}\right]^{T}, \quad A_{0}=$ $\left[\begin{array}{ccccccc}-k_{1} & g_{1} & 0 & \ldots & 0 & 0 & 0 \\ -k_{2} & 0 & g_{2} & \ldots & 0 & 0 & 0 \\ \vdots & & & & & & \\ -k_{n-1} & 0 & 0 & \ldots & 0 & g_{(n-1)} & 0 \\ -k_{n} & 0 & 0 & \ldots & 0 & 0 & g_{n} \\ 0 & 0 & 0 & \ldots & 0 & 0 & -\beta\end{array}\right], K=\left[k_{1}, \ldots, k_{n}, 0\right]^{T}$, and $\bar{F}=\left[\theta_{1}^{T} \varphi_{1}\left(x_{1}\right), \theta_{2}^{T} \varphi_{2}\left(\underline{x}_{2}\right), \ldots, \theta_{n}^{T} \varphi_{n}\left(\hat{x}_{n}\right), 0\right]^{T}$.

The observer gain matrix $K$ is given such that $A_{0}$ is a Hurwitz matrix. Therefore, for any chosen positive definite matrix $Q=Q^{T}>0$, there is a positive definite matrix $P=$ $P^{T}>0$ that satisfies

$$
A_{0}^{T} P+P A_{0}=-Q .
$$

The observer errors can be obtained as

$$
e=x-\widehat{x}=\left[e_{1}, \ldots, e_{n}\right]^{T} .
$$

From (12), (16), and (17), the observer error is

$$
\dot{e}=A_{0} e+d+\varepsilon+\Delta f+\widetilde{\Theta},
$$

where $\widetilde{\theta}_{i}=\theta_{i}^{*}-\theta_{i}$ and $\widetilde{\Theta}=\left[\widetilde{\theta}_{1}^{T} \varphi_{1}\left(x_{1}\right), \ldots, \widetilde{\theta}_{n}^{T} \varphi_{n}\left(\underline{\hat{x}}_{n}\right)\right]^{T}$.

Consider the Lyapunov function candidate as

$$
V_{0}=e^{T} P e .
$$

The time derivative of $V_{0}$ with (20) is

$$
\begin{aligned}
\dot{V}_{0}= & \dot{e}^{T} P e+e^{T} P \dot{e}, \\
= & \left(e^{T} A_{0}^{T}+d^{T}+\varepsilon^{T}+\Delta f^{T}+\widetilde{\Theta}^{T}\right) P e \\
& +e^{T} P\left[A_{0} e+d+\varepsilon+\Delta f+\widetilde{\Theta}\right], \\
= & e^{T}\left[A_{0}^{T} P+P A_{0}\right] e+2 e^{T} P[d+\varepsilon+\Delta f+\widetilde{\Theta}], \\
= & -e^{T} Q e+2 e^{T} P[d+\varepsilon+\Delta f+\widetilde{\Theta}] .
\end{aligned}
$$

By using Young's inequality and Assumptions 3-5, the inequalities can be obtained as

$$
\begin{gathered}
2 e^{T} P \varepsilon+2 e^{T} P d \leq 2\|e\|^{2}+\|P\|^{2}\left\|\varepsilon^{*}\right\|^{2}+\|P\|^{2}\left\|d^{*}\right\|^{2}, \\
2 e^{T} P \widetilde{\Theta} \leq\|e\|^{2}+\|P\|^{2} \sum_{l=1}^{n} \widetilde{\theta}_{l}^{T} \widetilde{\theta}_{l},
\end{gathered}
$$


$2 e^{T} P \Delta f \leq\|e\|^{2}+\|P\|^{2}\|\Delta f\|^{2} \leq\|e\|^{2}+\|P\|^{2}\left(\sum_{j=2}^{n} L_{j}^{2}\|e\|^{2}\right)=r_{0}\|e\|^{2}$,

where $r_{0}=1+\|P\|^{2} \sum_{j=2}^{n} L_{j}^{2}$.

Substituting (23)-(25) into (22) yields

$$
\begin{gathered}
\dot{V}_{0} \leq-e^{T} Q e+\left(r_{0}+3\right)\|e\|^{2}+\|P\|^{2}\left\|\varepsilon^{*}\right\|^{2} \\
+\|P\|^{2}\left\|d^{*}\right\|^{2}+\|P\|^{2} \sum_{l=1}^{n} \widetilde{\theta}_{l}^{T} \widetilde{\theta}_{l} .
\end{gathered}
$$

2.5. Tracking Error Transformation. A new variable parameter independent of the initial error is proposed, which satisfies the expected variable-parameter scheme tracking performance constraints. Then, the tracking error is defined as $E_{1}=y-y_{d}$. Predefined performance control constraint will be obtained as inequality holds for all $t \geq 0$ :

$$
-\iota_{d}(t) \rho(t)<E_{1}(t)<\iota_{u}(t) \rho(t),
$$

where smooth function $\iota_{d}(t)$ and $\iota_{u}(t)$ satisfies the follow properties. (1) $\iota_{d}(t)>0, \iota_{u}(t)>0$ and strictly decreasing. (2) $\lim _{t \longrightarrow 0} \iota_{d}(t)=+\infty ; \quad \lim _{t \longrightarrow \infty} \iota_{d}(t)=C_{1}, C_{1} \in R_{+}$; $\lim _{t \longrightarrow 0} l_{u}(t)=+\infty$; and $\lim _{t \longrightarrow \infty} l_{u}(t)=C_{2}, C_{2} \in R_{+}$.

In this article, $\iota_{d}(t)$ and $\iota_{u}(t)$ can be chosen as

$$
\left\{\begin{array}{l}
i_{d}(t)=-\lambda_{d} l_{d}(t)+h_{d}, \\
i_{u}(t)=-\lambda_{u} l_{u}(t)+h_{u},
\end{array}\right.
$$

where $\lambda_{d}, \lambda_{u}, h_{d}$, and $h_{u}$ are positive constants.

And $\rho(t)$ is an appropriate performance boundary function and is defined as $\rho(t)=\left(\rho(0)-\rho_{\infty}\right) e^{-\lambda t}+\rho_{\infty}$, which satisfies the following. (1) $\rho(t)$ is positive and strictly decreasing. (2) $\lim _{t \rightarrow \infty} \rho(t)=\rho_{\infty}>0$.

Integrating equality $(28)$ over $[0, t)$, we have

$$
\iota_{d}(t)=\left[\iota_{d}(0)-\frac{h_{d}}{\lambda_{d}}\right] e^{-\lambda_{d} t}+\frac{h_{d}}{\lambda_{d}} .
$$

In the same way,

$$
\iota_{u}(t)=\left[\iota_{u}(0)-\frac{h_{u}}{\lambda_{u}}\right] e^{-\lambda_{u} t}+\frac{h_{u}}{\lambda_{u}} .
$$

By the above analysis, $\iota_{d}(t)$ and $\iota_{u}(t)$ converge exponentially to constants $h_{d} / \lambda_{d}$ and $h_{u} / \lambda_{u}$, and the convergence rate can be improved by adding $\lambda_{d}$ and $\lambda_{u}$. When $\iota_{d}(t)$ and $\iota_{u}(t)$ converge to constant values, inequality constraint (27) is degenerated as follows:

$$
-\frac{h_{d}}{\lambda_{d}} \rho(t)<E_{1}(t)<\frac{h_{u}}{\lambda_{u}} \rho(t) .
$$

According to (31), when the system is stable, the upper bound of the steady-state error is $\max \left\{\left(h_{d} / \lambda_{d}\right)\right.$, $\left.\left(h_{u} / h_{u}\right)\right\} \rho(\infty)$, and the error convergence speed and the maximum overpass can be adjusted by the coefficient $\lambda_{d}, \lambda_{u}$, $h_{d}, h_{u}$, and $\rho(t)$.
The inequality constraint is transformed into equality constant, and the error transformation function $\phi\left(z, l_{d}, l_{u}\right)$ is defined as

$$
E_{1}(t)=\rho(t) \phi\left(z, \iota_{d}, \iota_{u}\right),
$$

where $z$ is transform error, and the continuous function $\phi\left(z, \iota_{\text {down }}, l_{\text {up }}\right)$ satisfies the following properties. (1) $\phi\left(z, \iota_{d}\right.$, $\left.\iota_{u}\right)$ is smooth and strictly increasing; $(2)-\iota_{d}(t)<$ $\phi\left(z, \iota_{d}, \iota_{u}\right)<\iota_{u}(t) ; \quad$ (3) $\lim _{z \longrightarrow-\infty} \phi\left(z, \iota_{d}, \iota_{u}\right)=-\iota_{d}(t)$; $\lim _{z \longrightarrow+\infty} \phi\left(z, \iota_{d}, \iota_{u}\right)=\iota_{u}(t)$.

By the properties of function $\phi\left(z, l_{d}, l_{u}\right)$, the inverse transformation is

$$
z_{1}=\phi^{-1}\left[\frac{E_{1}(t)}{\rho(t)}, \iota_{d}, \iota_{u}\right] .
$$

The error transformation function is defined as

$$
\phi\left[z, \iota_{d}, \iota_{u}\right]=\frac{\iota_{u} e^{z}-\iota_{d} e^{-z}}{e^{z}+e^{-z}} .
$$

Therefore, by (32), the boundless of the error transformation function $z_{1}$ results in the prescribed performance of the tracking error $E_{1}$.

Differentiating (33), it yields

$$
\dot{z}_{1}(t)=\frac{\partial \phi^{-1}}{\partial\left(E_{1} / \rho\right)} \frac{\partial\left(E_{1} / \rho\right)}{\partial t}=\frac{\partial \phi^{-1}}{\partial\left(E_{1} / \rho\right)}\left(\dot{E}_{1}-\frac{E_{1} \dot{\rho}}{\rho}\right) \text {. }
$$

Combining (35) and (12), one can obtain as

$$
\dot{z}_{1}=\omega\left(g_{1}(t) x_{2}+\theta_{1}^{* T} \varphi_{1}\left(x_{1}\right)+\varepsilon_{1}\left(x_{1}\right)+d_{1}\right)+D,
$$

where $\Phi=\left(\partial \phi^{-1}\right) /\left(\partial\left(E_{1} / \rho\right)\right) 1 / \rho>0$ and $D=\left(-\partial \phi^{-1}\right) /(\partial$ $\left.\left(E_{1} / \rho\right)\right) 1 / \rho\left(\dot{y}_{d}+E_{1} \dot{\rho} / \rho\right)$.

By derivation,

$$
\begin{aligned}
\dot{z}_{1} & =\frac{1}{2} \log \left(\frac{E_{1}}{\rho}+\iota_{u}\right)-\frac{1}{2} \log \left(\iota_{\mathrm{d}}-\frac{E_{1}}{\rho}\right), \\
\Phi & =\frac{1}{2 \rho}\left[\frac{1}{E_{1} / \rho+\iota_{d}}-\frac{1}{E_{1} / \rho-\iota_{u}}\right] .
\end{aligned}
$$

Remark 3. Note that the functions $\iota_{d}$ and $\iota_{u}$ are set to solve the problem of unknown initial error $E(0)$. In the predefined performance control of [34], the initial error is assumed to be known, but in the actual control, the exact value of the initial error is often not obtained, which limits the use of the predefined performance control. The reason why the initial values of $\iota_{d}(t)$ and $\iota_{u}(t)$ are set to infinity is mainly to guarantee $E(0) \in\left(-l_{d}, \iota_{u}\right)$; however, $\iota_{d}(0)$ and $\iota_{u}(0)$ just need to be set to a sufficiently large constant in the actual design. The facts justify this treatment.

\section{Control Design and Stability Analysis}

In this section, by utilizing adaptive backstepping technique and Lyapunov stability theorem, an observer-based adaptive fuzzy predefined performance decentralized control approach is developed. 
The coordinate changes are as follows:

$$
\left\{\begin{array}{l}
E_{1}=y-y_{d} \\
E_{i}=\hat{x}_{i}-\bar{\alpha}_{i}(i=2, \ldots, n-1) \\
E_{n}=\widehat{x}_{n}-\bar{\alpha}_{n}+\hat{x}_{n+1} \frac{g_{n}}{\beta} \\
\xi_{i}=\bar{\alpha}_{i}-\alpha_{i-1},
\end{array}\right.
$$

where $\alpha_{i}$ is the virtual control law and $\bar{\alpha}_{i}$ and $\xi_{i}$ are the filter signal and filter error of the first-order filter, respectively. Define a first-order filter as

$$
\begin{array}{r}
\zeta_{i} \dot{\bar{\alpha}}_{i}+\bar{\alpha}_{i}=\alpha_{i-1}, \\
\bar{\alpha}_{i}(0)=\alpha_{i-1}(0), \quad i=2, \ldots, n,
\end{array}
$$

where $\zeta_{i}$ is the design constant.

Step 1. By substituting (38) into (36), one can obtain as

$$
\dot{z}_{1}=\omega\left(g_{1}\left(E_{2}+\xi_{2}+\alpha_{1}+e_{2}\right)+\theta_{1}^{*} \varphi_{1}\left(x_{1}\right)+\varepsilon_{1}\left(x_{1}\right)+d_{1}\right)+D .
$$

Consider the Lyapunov function candidate as

$$
V_{1}=V_{0}+\frac{1}{2} z_{1}^{2}+\frac{1}{2 v_{1}}{\widetilde{\theta_{1}}}^{T} \tilde{\theta}_{1}
$$

where $v_{1}>0$ is a design constant. The time derivative of $V_{1}$ and substitute (40) and (26) into (41) as

$$
\begin{aligned}
\dot{V}_{1}= & \dot{V}_{0}+z_{1} \dot{z}_{1}+\frac{1}{v_{1}} \tilde{\theta}_{1}^{T} \dot{\tilde{\theta}}_{1} \leq-e^{T} Q e+\left(r_{0}+3\right)\|e\|^{2} \\
& +\|P\|^{2}\left\|\mathcal{E}^{*}\right\|^{2}+\|P\|^{2}\left\|d^{*}\right\|^{2}+\|P\|^{2} \sum_{l=1}^{n} \widetilde{\theta}_{l}^{T} \widetilde{\theta}_{l}+\theta_{1}^{*} \varphi_{1}\left(x_{1}\right) \\
& +z_{1}\left[\omega\left(g_{1}\left(E_{2}+\xi_{2}+\alpha_{1}+e_{2}\right)+\varepsilon_{1}\left(x_{1}\right)+d_{1}\right)+D\right] \\
& +\frac{1}{v_{1}} \tilde{\theta}_{1}^{T} \dot{\tilde{\theta}}_{1} .
\end{aligned}
$$

By using Young's inequality,

$$
\begin{aligned}
z_{1} \bowtie g_{1}\left(E_{2}+\xi_{2}\right) & \leq \omega^{2} z_{1}^{2}+\frac{1}{2} g_{1}^{* 2}\left(E_{2}^{2}+\xi_{2}^{2}\right), \\
z_{1} \bowtie g_{1} e_{2} & \leq \frac{1}{2} \oplus^{2} z_{1}^{2}+\frac{1}{2} g_{1}^{* 2}\|e\|^{2}, \\
z_{1} \oplus \varepsilon_{1} & \leq \frac{1}{2} \oplus^{2} z_{1}^{2}+\frac{1}{2} \varepsilon_{1}^{2}, \\
z_{1} \oplus d_{1} & \leq \frac{1}{2} \omega^{2} z_{1}^{2}+\frac{1}{2} d_{1}^{2} .
\end{aligned}
$$

From (43)-(46), one can obtain as

$$
\begin{aligned}
\dot{V}_{1} \leq & -\left(\lambda_{\min }(Q)-r_{0}-3-\frac{1}{2} g_{1}^{* 2}\right)\|e\|^{2}+\|P\|^{2}\left\|\varepsilon^{*}\right\|^{2} \\
& +\|P\|^{2}\left\|d^{*}\right\|^{2}+\|P\|^{2} \sum_{l=1}^{n} \widetilde{\theta}_{l}^{T} \tilde{\theta}_{l} \\
& +z_{1}\left[\Phi\left(\frac{5}{2} \bowtie z_{1}+g_{1} \alpha_{1}+\theta_{1}^{T} \varphi_{1}\left(x_{1}\right)\right)+D\right] \\
& +\frac{1}{2} \varepsilon_{1}^{* 2}+\frac{1}{2} d_{1}^{* 2}+\frac{1}{2} g_{1}^{* 2}\left(E_{2}^{2}+\xi_{2}^{2}\right) \\
& +\frac{1}{v_{1}} \tilde{\theta}_{1}^{T}\left(v_{1} \varphi_{1}\left(x_{1}\right) z_{1} \Phi-\dot{\theta}_{1}\right) .
\end{aligned}
$$

Select the virtual control function $\alpha_{1}$ and the adaptive laws $\theta_{1}$ as

$$
\alpha_{1}=N\left(\mu_{1}\right)\left[\omega_{1} z_{1}+\frac{5}{2} \Phi z_{1}+\theta_{1}^{T} \varphi_{1}\left(x_{1}\right)+\frac{D}{\varpi}\right],
$$

$$
\dot{\mu}_{1}=z_{1} \bowtie\left[\omega_{1} z_{1}+\frac{5}{2} \bowtie z_{1}+\theta_{1}^{T} \varphi_{1}\left(x_{1}\right)+\frac{D}{\varpi}\right]
$$

$$
\dot{\theta}_{1}=v_{1} \varphi_{1}\left(x_{1}\right) z_{1} \omega-\sigma \theta_{1}
$$

where $\omega_{1}>0$ and $\sigma>0$ are design constants.

Substituting (48)-(50) into (47) yields

$$
\begin{aligned}
\dot{V}_{1} \leq & -r_{1}\|e\|^{2}+\|P\|^{2}\left\|\mathcal{E}^{*}\right\|^{2}+\|P\|^{2}\left\|d^{*}\right\|^{2}+\|P\|^{2} \sum_{l=1}^{n} \tilde{\theta}_{l}^{T} \tilde{\theta}_{l} \\
& -\omega_{1} \oplus z_{1}^{2}+\frac{\sigma}{v_{1}} \tilde{\theta}_{1}^{T} \theta_{1}+\frac{1}{2} g_{1}^{* 2}\left(E_{2}^{2}+\xi_{2}^{2}\right) \\
& +\left(g N\left(\mu_{1}\right)+1\right) \dot{\mu}_{1}+\frac{1}{2} \varepsilon_{1}^{* 2}+\frac{1}{2} d_{1}^{* 2},
\end{aligned}
$$

where $r_{1}=\lambda_{\min }(Q)-r_{0}-3-(1 / 2) g_{1}^{* 2}$

By using Young's inequality,

$$
\frac{\sigma}{v_{1}} \widetilde{\theta}_{1}^{T} \theta_{1}=\frac{\sigma}{v_{1}} \widetilde{\theta}_{1}^{T}\left(-\widetilde{\theta}_{1}+\theta_{1}^{*}\right) \leq-\frac{\sigma}{2 v_{1}} \widetilde{\theta}_{1}^{T} \widetilde{\theta}_{1}+\frac{\sigma}{2 v_{1}} \theta_{1}^{* T} \theta_{1}^{*} \text {. }
$$

Substitute (52) into (51) as follows:

$$
\begin{aligned}
\dot{V}_{1} \leq & -r_{1}\|e\|^{2}+\|P\|^{2} \sum_{l=1}^{n} \widetilde{\theta}_{l}^{T} \widetilde{\theta}_{l}-\omega_{1} \bowtie z_{1}^{2}-\frac{\sigma}{2 v_{1}} \widetilde{\theta}_{1}^{T} \widetilde{\theta}_{1} \\
& +\frac{1}{2} g_{1}^{* 2}\left(E_{2}^{2}+\xi_{2}^{2}\right)+\left(g N\left(\mu_{1}\right)+1\right) \dot{\mu}_{1}+M_{1},
\end{aligned}
$$

where $\quad M_{1}=\|P\|^{2}\left\|\varepsilon^{*}\right\|^{2}+\|P\|^{2}\left\|d^{*}\right\|^{2}+(1 / 2) \varepsilon_{1}^{* 2}+(1 / 2)$ $d_{1}^{* 2}+\left(\sigma / 2 v_{1}\right) \theta_{1}^{* T} \theta_{1}^{*}$.

Step $2(i(i=2, \ldots, n-1))$. The time derivative of $E_{i}$ is 


$$
\begin{aligned}
\dot{E}_{i} & =\dot{\hat{x}}_{i}-\dot{\bar{\alpha}}_{i}=g_{i} \widehat{x}_{i+1}+\theta_{i}^{* T} \varphi_{i}\left(\hat{\underline{x}}_{i}\right)+\varepsilon_{i}+k_{i} e_{1}-\dot{\bar{\alpha}}_{i} \\
& =g_{i}\left(E_{i+1}+\xi_{i+1}+\alpha_{i}\right)+\theta_{i}^{* T} \varphi_{i}\left(\underline{\underline{x}}_{i}\right)+\varepsilon_{i}+k_{i} e_{1}-\dot{\bar{\alpha}}_{i} .
\end{aligned}
$$

Consider the Lyapunov function candidate as

$$
V_{i}=V_{i-1}+\frac{1}{2} E_{i}^{2}+\frac{1}{2 v_{i}} \tilde{\theta}_{i}^{T} \widetilde{\theta}_{i}+\frac{1}{2} \xi_{i}^{2}
$$

where $v_{i}>0$ is a design constant. The derivative of $V_{i}$ with time is

$$
\begin{aligned}
\dot{V}_{i}= & \dot{V}_{i-1}+E_{i} \dot{E}_{i}+\frac{1}{v_{i}} \tilde{\theta}_{i}^{T} \dot{\tilde{\theta}}_{i}+\xi_{i} \dot{\xi}_{i} \leq-r_{1}\|e\|^{2}+\|P\|^{2} \sum_{l=1}^{n} \tilde{\theta}_{l}^{T} \widetilde{\theta}_{l} \\
& -\omega_{1} \Phi z_{1}^{2}-\sum_{l=1}^{i-1} \frac{\sigma}{2 v_{l}} \tilde{\theta}_{l}^{T} \widetilde{\theta}_{l}+\frac{1}{2} \sum_{l=1}^{i-1} g_{l}^{* 2}\left(E_{l+1}^{2}+\xi_{l+1}^{2}\right) \\
& +M_{i-1}-\sum_{l=2}^{i-1} \omega_{l} E_{l}^{2}+E_{i}\left[g_{i}\left(E_{i+1}+\xi_{i+1}+\alpha_{i}\right)\right. \\
& \left.+\theta_{i}^{* T} \varphi_{i}\left(\underline{x}_{i}\right)+\varepsilon_{i}+k_{i} e_{1}-\dot{\bar{\alpha}}_{i}\right]+\frac{1}{v_{i}} \widetilde{\theta}_{i}^{T} \dot{\tilde{\theta}}_{i}+\xi_{i} \dot{\xi}_{i} \\
& +\sum_{l=1}^{i-1}\left(g N\left(\mu_{l}\right)+1\right) \dot{\mu}_{l},
\end{aligned}
$$

where $M_{i-1}=M_{i-2}+(1 / 2) \varepsilon_{i-1}^{* 2}+\left(\sigma / 2 v_{i-1}\right) \theta_{i-1}^{* T} \theta_{i-1}^{*}$.

By using Young's inequality,

$$
\begin{aligned}
E_{i} g_{i}\left(E_{i+1}+\xi_{i+1}\right) & \leq E_{i}^{2}+\frac{1}{2} g_{i}^{* 2}\left(E_{i+1}^{2}+\xi_{i+1}^{2}\right), \\
E_{i} \varepsilon_{i} & \leq \frac{1}{2} E_{i}^{2}+\frac{1}{2} \varepsilon_{i}^{* 2} .
\end{aligned}
$$

From (57) and (58), one can obtain as

$$
\begin{aligned}
\dot{V}_{i} \leq & -r_{1}\|e\|^{2}+\|P\|^{2} \sum_{l=1}^{n} \widetilde{\theta}_{l}^{T} \widetilde{\theta}_{l}-\omega_{1} \omega z_{1}^{2}-\sum_{l=1}^{i-1} \frac{\sigma}{2 v_{l}} \tilde{\theta}_{l}^{T} \widetilde{\theta}_{l} \\
& +\frac{1}{2} \sum_{l=1}^{i-1} g_{l}^{* 2}\left(E_{l+1}^{2}+\xi_{l+1}^{2}\right)+M_{i-1}-\sum_{l=2}^{i-1} \omega_{l} E_{l}^{2} \\
& +E_{i}\left[g_{i} \alpha_{i}+\theta_{i}^{T} \varphi_{i}\left(\underline{x}_{i}\right)+\frac{3}{2} E_{i}+k_{i} e_{1}-\dot{\bar{\alpha}}_{i}\right] \\
& +\frac{1}{2} g_{i}^{* 2}\left(E_{i+1}^{2}+\xi_{i+1}^{2}\right)+\frac{1}{2} \varepsilon_{i}^{* 2}+\frac{1}{v_{i}} \widetilde{\theta}_{i}^{T}\left(v_{i} E_{i} \varphi_{i}\left(\underline{x}_{i}\right)-\dot{\theta}_{i}\right) \\
& +\xi_{i} \dot{\xi}_{i}+\sum_{l=1}^{i-1}\left(g N\left(\mu_{l}\right)+1\right) \dot{\mu}_{l} .
\end{aligned}
$$

Select the virtual control function $\alpha_{i}$ and the adaptive laws $\theta_{i}$ as

$$
\begin{aligned}
& \alpha_{i}=N\left(\mu_{i}\right)\left[\omega_{i} E_{i}+\frac{3}{2} E_{i}+\theta_{i}^{T} \varphi_{i}\left(\hat{\underline{x}}_{i}\right)+k_{i} e_{1}-\dot{\bar{\alpha}}_{i}\right], \\
& \dot{\mu}_{i}=E_{i}\left[\omega_{i} E_{i}+\frac{3}{2} E_{i}+\theta_{i}^{T} \varphi_{i}\left(\widehat{\underline{x}}_{i}\right)+k_{i} e_{1}-\dot{\bar{\alpha}}_{i}\right], \\
& \dot{\theta}_{i}=v_{i} E_{i} \varphi_{i}\left(\hat{x}_{i}\right)-\sigma \theta_{i} .
\end{aligned}
$$

Substituting (60)-(62) into (59) yields

$$
\begin{aligned}
\dot{V}_{i} \leq & -r_{1}\|e\|^{2}+\|P\|^{2} \sum_{l=1}^{n} \widetilde{\theta}_{l}^{T} \widetilde{\theta}_{l}-\omega_{1} \oplus z_{1}^{2}-\sum_{l=1}^{i-1} \frac{\sigma}{2 v_{l}} \widetilde{\theta}_{l}^{T} \widetilde{\theta}_{l} \\
& +\frac{1}{2} \sum_{l=1}^{i} g_{l}^{* 2}\left(E_{l+1}^{2}+\xi_{l+1}^{2}\right)+M_{i}+\sum_{l=1}^{i} \xi_{l} \dot{\xi}_{l}-\sum_{l=2}^{i} \omega_{l} E_{l}^{2} \\
& +\sum_{l=1}^{i}\left(g N\left(\mu_{l}\right)+1\right) \dot{\mu}_{l}+M_{i-1}+\frac{1}{2} \varepsilon_{i}^{* 2}+\frac{\sigma}{v_{i}} \widetilde{\theta}_{i}^{T} \theta_{i} .
\end{aligned}
$$

According to the definition of $\alpha_{1}, \alpha_{2}$, and $\alpha_{i}$, one can get $\dot{\alpha}_{i}=\eta_{i}\left(E_{1}, \ldots, E_{i}, E_{i+1}, \theta_{1}, \ldots, \theta_{i}, y_{d}, \dot{y}_{d}, \ddot{y}_{d}, \xi_{2}, \ldots, \xi_{i}\right)$, where $\eta_{i}$ is a continuous function. Given that any $\psi, C_{i}=$ $\left\{\left(E_{1}, \ldots, E_{i}, E_{i+1}, \theta_{1}, \ldots, \theta_{i}, y_{d}, \dot{y}_{d}, \ddot{y}_{d}, \xi_{2}, \ldots, \xi_{i}\right)^{T}: \vartheta<\psi\right\}$ is a prefixed compact set, where the compact set $C_{i}$ can be made larger as needed. Therefore, the maximum value of the continuous function $\eta_{i}$ is $B_{i+1}$ on $C_{i} * \Delta_{0}$ based on Assumption 1 of the compact set $\Upsilon$ and the compact set $C_{i}$. It is obvious that

$$
\begin{aligned}
\dot{\xi}_{i} & =-\frac{\xi_{i}}{\chi_{i}}+\eta_{i-1}\left(E_{1}, \ldots, E_{i}, \theta_{1}, \ldots, \theta_{i-1}, y_{d}, \dot{y}_{d}, \ddot{y}_{d}, \xi_{2}, \ldots, \xi_{i-1}\right) \\
& \leq-\frac{\xi_{i}}{\chi_{i}}+B_{i} .
\end{aligned}
$$

Utilising Young's inequality,

$$
\begin{aligned}
& \frac{\sigma}{v_{i}} \widetilde{\theta}_{i}^{T} \theta_{i}=\frac{\sigma}{v_{i}} \widetilde{\theta}_{i}^{T}\left(-\widetilde{\theta}_{i}+\theta_{i}^{*}\right) \leq-\frac{\sigma}{2 v_{i}} \widetilde{\theta}_{i}^{T} \widetilde{\theta}_{i}+\frac{\sigma}{2 v_{i}} \theta_{i}^{* T} \theta_{i}^{*}, \\
& \xi_{i} \dot{\xi}_{i} \leq \xi_{i}\left[-\frac{\xi_{i}}{\chi_{i}}+B_{i}\right] \leq-\frac{\xi_{i}^{2}}{\chi_{i}}+\xi_{i} B_{i} \leq-\left[\frac{1}{\chi_{i}}-\frac{B_{i}^{2}}{2 \pi_{i}}\right] \xi_{i}^{2}+\frac{\pi_{i}}{2},
\end{aligned}
$$

where $\pi_{i}$ is the design constant.

Substitute (65) and (66) into (63) as follows:

$$
\begin{aligned}
\dot{V}_{i} \leq & -r_{1}\|e\|^{2}+\|P\|^{2} \sum_{l=1}^{n} \tilde{\theta}_{l}^{T} \tilde{\theta}_{l}-\omega_{1} \bowtie z_{1}^{2}-\sum_{l=1}^{i} \frac{\sigma}{2 v_{l}} \tilde{\theta}_{l}^{T} \widetilde{\theta}_{l} \\
& +\frac{1}{2} \sum_{l=1}^{i} g_{l}^{* 2}\left(E_{l+1}^{2}+\xi_{l+1}^{2}\right)-\sum_{l=1}^{i}\left[\frac{1}{\chi_{i}}-\frac{B_{i}^{2}}{2 \pi_{i}}\right] \xi_{i}^{2} \\
& -\sum_{l=2}^{i} \omega_{l} E_{l}^{2}+\sum_{l=1}^{i}\left(g N\left(\mu_{l}\right)+1\right) \dot{\mu}_{l}+M_{i},
\end{aligned}
$$


where $M_{i}=M_{i-1}+(1 / 2) \varepsilon_{i}^{* 2}+\left(\sigma / 2 v_{i}\right) \theta_{i}^{* T} \theta_{i}^{*}+\left(\pi_{i} / 2\right)$.

Step $3 \mathrm{n}$. The time derivative of $E_{n}$ is

$$
\begin{aligned}
\dot{E}_{n}= & \dot{\hat{x}}_{n}-\dot{\bar{\alpha}}_{n}+\dot{\hat{x}}_{n+1} \frac{g_{n}}{\beta}=g_{n}\left(\widehat{x}_{n+1}-u\right)+\theta_{n}^{* T} \varphi_{n}\left(\underline{\hat{x}}_{n}\right) \\
& +\varepsilon_{n}+k_{n} e_{1}-\dot{\bar{\alpha}}_{n}+\frac{g_{n}}{\beta}\left(-\beta \widehat{x}_{n+1}+2 \beta u\right) \\
= & g_{n} u+\theta_{n}^{* T} \varphi_{n}\left(\underline{\hat{x}}_{n}\right)+\varepsilon_{n}+k_{n} e_{1}-\dot{\bar{\alpha}}_{n} .
\end{aligned}
$$

Consider the Lyapunov function candidate as

$$
V_{n}=V_{n-1}+\frac{1}{2} E_{n}^{2}+\frac{1}{2 v_{n}} \widetilde{\theta}_{n}^{T} \widetilde{\theta}_{n}+\frac{1}{2} \xi_{n}^{2}
$$

where $v_{n}>0$ is a design constant. The derivative of $V_{n}$ with time along with (68) is

$$
\begin{aligned}
\dot{V}_{n}= & \dot{V}_{n-1}+E_{n} \dot{E}_{n}+\frac{1}{v_{n}} \widetilde{\theta}_{n}^{T} \dot{\tilde{\theta}}_{n}+\xi_{n} \dot{\xi}_{n} \\
= & \dot{V}_{n-1}+E_{n}\left[g_{n} u+\theta_{n}^{* T} \varphi_{n}\left(\underline{\underline{x}}_{n}\right)+\varepsilon_{n}+k_{n} e_{1}-\dot{\bar{\alpha}}_{n}\right] \\
& +\frac{1}{v_{n}} \widetilde{\theta}_{n}^{T} \dot{\tilde{\theta}}_{n}+\xi_{n} \dot{\xi}_{n} .
\end{aligned}
$$

By using Young's inequality,

$$
E_{n} \varepsilon_{n} \leq \frac{1}{2} E_{n}^{2}+\frac{1}{2} \varepsilon_{n}^{* 2} .
$$

From (71), one can obtain

$$
\begin{aligned}
\dot{V}_{n} \leq & \dot{V}_{n-1}+E_{n}\left[g_{n} u+\theta_{n}^{T} \varphi_{n}\left(\underline{x}_{n}\right)+\frac{1}{2} E_{n}+k_{n} e_{1}-\dot{\bar{\alpha}}_{n}\right] \\
& +\frac{1}{2} \varepsilon_{n}^{* 2}+\frac{1}{v_{n}} \tilde{\theta}_{n}^{T}\left(v_{n} E_{n} \varphi_{n}\left(\underline{x}_{n}\right)-\dot{\theta}_{n}\right)+\xi_{n} \dot{\xi}_{n} .
\end{aligned}
$$

Select the actual control input $u$ and the adaptive laws $\theta_{n}$ as

$$
\begin{aligned}
& u=N\left(\mu_{n}\right)\left[\omega_{n} E_{n}+\frac{1}{2} E_{n}+\theta_{n}^{T} \varphi_{n}\left(\underline{\hat{x}}_{n}\right)+k_{n} e_{1}-\dot{\bar{\alpha}}_{n}\right], \\
& \dot{\mu}_{n}=E_{n}\left[\omega_{n} E_{n}+\frac{1}{2} E_{n}+\theta_{n}^{T} \varphi_{n}\left(\underline{\hat{x}}_{n}\right)+k_{n} e_{1}-\dot{\bar{\alpha}}_{n}\right], \\
& \dot{\theta}_{n}=v_{n} E_{n} \varphi_{n}\left(\hat{x}_{n}\right)-\sigma \theta_{n} .
\end{aligned}
$$

Substituting (73)-(75) into (72) yields

$$
\begin{aligned}
\dot{V}_{n} \leq & -r_{1}\|e\|^{2}+\|P\|^{2} \sum_{l=1}^{n} \widetilde{\theta}_{l}^{T} \widetilde{\theta}_{l}-\omega_{1} \Phi z_{1}^{2}-\sum_{l=1}^{n-1} \frac{\sigma}{2 v_{l}} \widetilde{\theta}_{l}^{T} \widetilde{\theta}_{l} \\
& +\frac{1}{2} \sum_{l=1}^{n-1} g_{l}^{* 2}\left(E_{l+1}^{2}+\xi_{l+1}^{2}\right)+\sum_{l=1}^{n} \xi_{l} \dot{\xi}_{l}-\sum_{l=2}^{n} \omega_{l} E_{l}^{2} \\
& +\sum_{l=1}^{n}\left(g N\left(\mu_{l}\right)+1\right) \dot{\mu}_{l}+M_{n-1}+\frac{1}{2} \varepsilon_{n}^{* 2}+\frac{\sigma}{v_{n}} \tilde{\theta}_{n}^{T} \theta_{n} .
\end{aligned}
$$

Combining Young's inequality and inequality (64), one can obtain

$$
\begin{aligned}
\frac{\sigma}{v_{n}} \widetilde{\theta}_{n}^{T} \theta_{n} & =\frac{\sigma}{v_{n}} \tilde{\theta}_{n}^{T}\left(-\widetilde{\theta}_{n}+\theta_{n}^{*}\right) \leq-\frac{\sigma}{2 v_{n}} \widetilde{\theta}_{n}^{T} \widetilde{\theta}_{n}+\frac{\sigma}{2 v_{n}} \theta_{n}^{* T} \theta_{n}^{*}, \\
\xi_{n} \dot{\xi}_{n} & \leq-\frac{\xi_{n}^{2}}{\chi_{n}}+\xi_{n} B_{n} \leq-\left[\frac{1}{\chi_{n}}-\frac{B_{n}^{2}}{2 \pi_{n}}\right] \xi_{n}^{2}+\frac{\pi_{n}}{2} .
\end{aligned}
$$

Substitute (77) and (78) into (76) as follows

$$
\begin{aligned}
\dot{V}_{n} \leq & -r_{1}\|e\|^{2}+\|P\|^{2} \sum_{l=1}^{n} \widetilde{\theta}_{l}^{T} \widetilde{\theta}_{l}-\omega_{1} \oplus z_{1}^{2}-\sum_{l=1}^{n} \frac{\sigma}{2 v_{l}} \widetilde{\theta}_{l}^{T} \widetilde{\theta}_{l} \\
& +\frac{1}{2} \sum_{l=1}^{n-1} g_{l}^{* 2}\left(E_{l+1}^{2}+\xi_{l+1}^{2}\right)+M_{n}-\sum_{l=1}^{n}\left[\frac{1}{\chi_{n}}-\frac{B_{n}^{2}}{2 \pi_{n}}\right] \xi_{n}^{2} \\
& -\sum_{l=2}^{n} \omega_{l} E_{l}^{2}+\sum_{l=1}^{n}\left(g N\left(\mu_{l}\right)+1\right) \dot{\mu}_{l},
\end{aligned}
$$

where $M_{n}=M_{n-1}+(1 / 2) \varepsilon_{n}^{* 2}+\left(\pi_{n} / 2\right)+\left(\sigma / 2 v_{l}\right) \theta_{l}^{* T} \theta_{l}^{*}$. The following theorem is summarized by the above controller design and stability analysis.

Theorem 1. Consider a class of nonlinear pure-feedback systems (1), with uncertain functions, unmeasurable states, and input delay, and the state observer is designed as (16). Under Assumptions1-5, the adaptive laws are designed as (48), (60), and (73). The virtual control functions are chosen as (50) and (62). And the actual control input function (75) can guarantee all the signals in the closed-loop system are SGUUB. Meanwhile, the tracking error does not deviate from the prescribed performance bound (31), and the observer errors converge within a small zero region.

Proof. Let

$$
\begin{array}{r}
\bar{c}=\min \left\{\frac{2 r_{1}}{\lambda_{\min }(P)}, 2 v_{l}\left(\frac{\sigma}{2 v_{l}}-\|P\|^{2}\right), 2 \bowtie \omega_{1}, 2 \omega_{k}, 2\left[\frac{1}{\chi_{n}}-\frac{B_{n}^{2}}{2 \pi_{n}}\right]\right\}, \\
k=2, \ldots, n ; l=1,2, \ldots, n,
\end{array}
$$

where $\bar{c}$ will be positive by choosing appropriate parameters.

Then, (79) can be finally expressed as

$$
\dot{V}_{n} \leq-\bar{c} V_{n}+\sum_{l=1}^{n}\left(g N\left(\mu_{l}\right)+1\right) \dot{\mu}_{l}+\frac{1}{2} \sum_{l=1}^{n-1} g_{l}^{* 2}\left(E_{l+1}^{2}+\xi_{l+1}^{2}\right)+M_{n}
$$

where $\quad M_{n}=\|P\|^{2}\left\|\varepsilon^{*}\right\|^{2}+\|P\|^{2}\left\|d^{*}\right\|^{2}+\sum_{l=1}^{n}(1 / 2) \varepsilon_{l}^{* 2}+\sum_{l=i}^{n}$ $\left(\pi_{l} / 2\right)+\sum_{l=1}^{n}\left(\sigma / 2 v_{l}\right) \theta_{l}^{* T} \theta_{l}^{*}$ is bounded.

Multiplying both sides of (81) by $e^{-\bar{c} t}$ generates 


$$
\begin{aligned}
\frac{\mathrm{d}\left(V_{n} e^{-\bar{c} t}\right)}{\mathrm{d} t} \leq & \sum_{l=1}^{n}\left(g N\left(\mu_{l}\right)+1\right) \dot{\mu}_{l} e^{-\bar{c} t}+M_{n} e^{-\bar{c} t} \\
& +\frac{1}{2} \sum_{l=1}^{n-1} g_{l}^{* 2}\left(E_{l+1}^{2}+\xi_{l+1}^{2}\right) e^{-\bar{c} t} .
\end{aligned}
$$

Integrating the above inequality over $\left[0, t_{f}\right)$ and then multiplying both sides by $e^{-\bar{c} t}$ yields

$$
\begin{aligned}
V_{n} \leq & V(0) e^{-\bar{c} t}+\frac{M_{n+1}}{\bar{c}}\left(1-e^{-\bar{c} t}\right) \\
& +e^{-\bar{c} t} \int_{0}^{t} \sum_{l=1}^{n}\left(g N\left(\mu_{l}\right)+1\right) \dot{\mu}_{l} e^{-\bar{c} \tau} \mathrm{d} \tau \\
& +e^{-\bar{c} t} \int_{0}^{t} \frac{1}{2} \sum_{l=1}^{n-1} g_{l}^{* 2}\left(E_{l+1}^{2}+\xi_{l+1}^{2}\right) e^{-\bar{c} \tau} \mathrm{d} \tau .
\end{aligned}
$$

Based on Lemma 2, $V_{n}$ and $\mu$ can be proved to be bounded. In addition, since $E_{l+1}$ and $\xi_{l+1}$ are semiglobal and ultimately uniformly bounded, $e^{-\bar{c} t} \int_{0}^{t} 1 / 2 \sum_{l=1}^{n-1} g_{l}^{* 2}$ $\left(E_{l+1}^{2}+\xi_{l+1}^{2}\right) e^{-\bar{c} \tau} \mathrm{d} \tau$ is bounded. Therefore, the stability of the whole closed-loop system is demonstrated. Furthermore, since $z_{1}$ can be proved to be uniformly bounded, the prescribed performance tracking control is achieved.

\section{Simulation Example}

Consider a class of nonlinear pure-feedback systems with unavailable states, unknown control direction, and input delay:

$$
\left\{\begin{array}{l}
\dot{x}_{1}=f_{1}\left(x_{1}, x_{2}\right)+d_{1}(t), \\
\dot{x}_{2}=f_{2}\left(\underline{x}_{2}, u(t-\delta)\right)+d_{2}(t), \\
y=x_{1}
\end{array}\right.
$$

where $x_{1}$ and $x_{2}$ are the system states and $u$ and $y$ are the system input and output, respectively. The smooth functions are used as $f_{1}\left(x_{1}, x_{2}\right)=x_{1}+x_{1} x_{2}$ and $f\left(\underline{x}_{2}, u(t-\delta)\right)=$ $\left(2+\sin \left(x_{1} x_{2}\right)\right)(1+u(t-\delta))$, and the external disturbances in this simulation are given as $d_{1}(t)=0.02 \cos (t)$ and $d_{2}(t)=0.03 \sin (t)$. The input delay is chosen as $\delta=0.01$, and the reference signal is given as $y_{d}=\sin (t)$.

The parameters in control functions and adaptive laws are given as $\omega_{1}=0.1, \omega_{2}=40, v_{1}=v_{2}=5$, and $\sigma_{1}=\sigma_{2}=20$. Parameter in a first-order filter is $\zeta_{2}=0.05$. As for the state observer, the observer gains are selected as $K=\left[k_{1}, k_{2}\right]^{T}=[10,100]^{T}$. In predefined performance controls, $\quad \iota_{u}(0)=\iota_{d}(0)=30, \quad \lambda_{d}=\lambda_{u}=0.8, \quad \lambda=2$, $h_{u}=h_{d}=0.4$, and $\rho_{\infty}=0.4$. The initial value of the system states are $x_{1}(0)=0.3$ and $x_{2}(0)=0.5$, and the initial values for the other parameters are zero.

The simulation results are shown in Figures 1-7, where the red and blue lines represent the approach proposed in

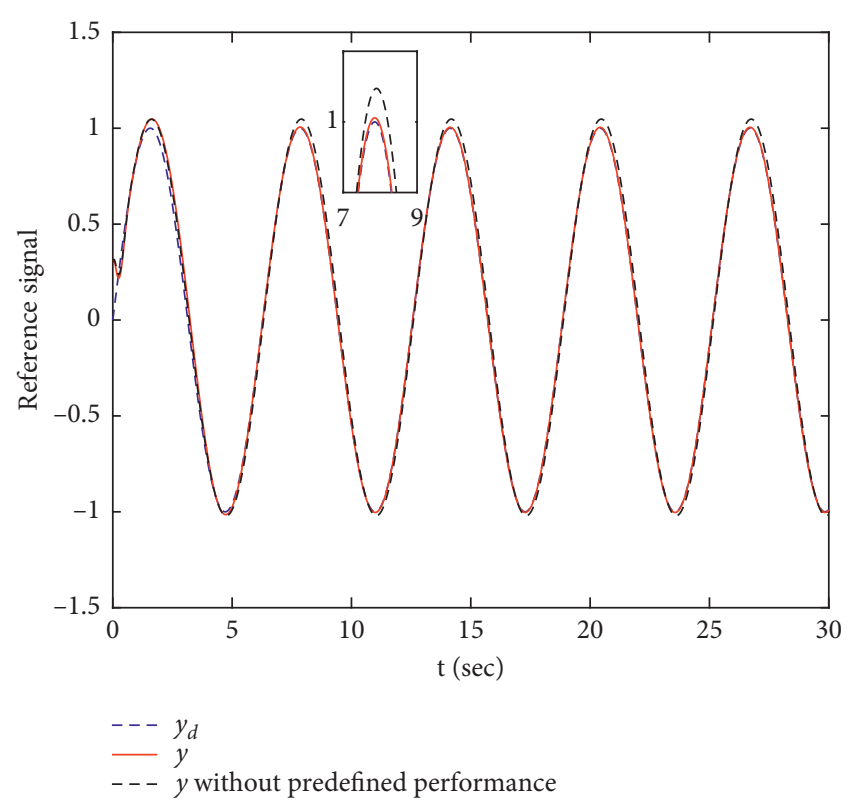

FIgURE 1: Trajectories of $y$ and $y_{d}$.

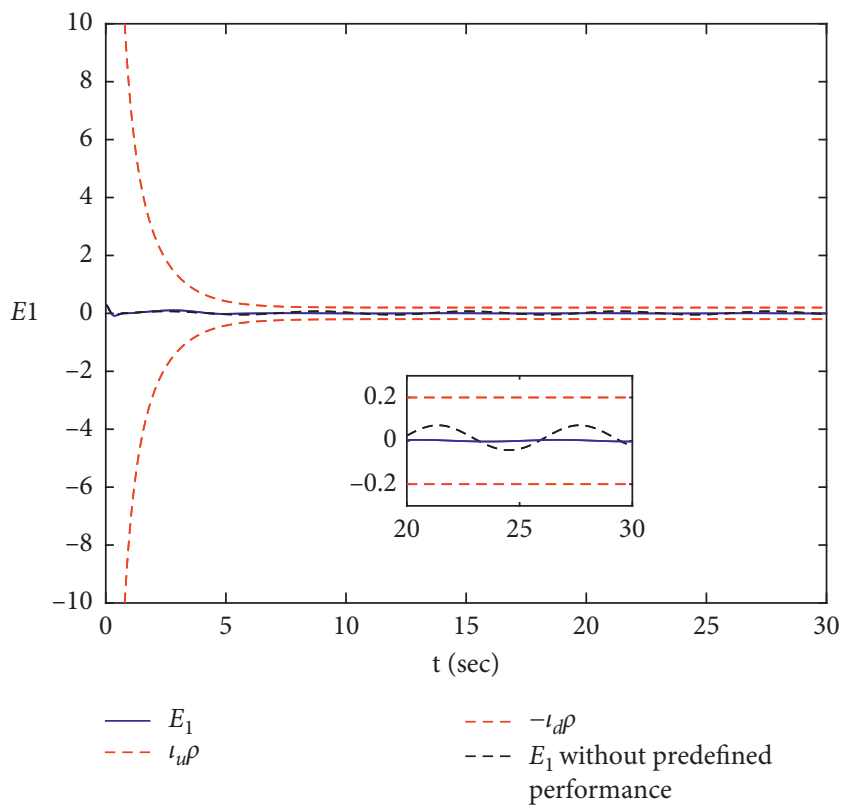

FIgURE 2: Tracking error $E_{1}$ with prescribed performance $\iota_{u} \rho$ and $-l_{d} \rho$.

this article, and the black line represents the removal of the variable-parameter predefined performance. Figure 1 shows the output trajectories of $y$ and the expected output signal $y_{d}$. The output tracking error and the prescribed performance boundaries are shown in Figures 2, and Figures 3 and 5 illustrate the trajectories of system states $x_{1}$ and $x_{2}$ and their estimates $\hat{x}_{1}$ and $\hat{x}_{2}$, respectively. The Tracking errors $e_{1}$ and $e_{2}$ of state $x_{1}$ and $x_{2}$ are shown in Figures 4 , and Figures 6 and 7 show the trajectories of the actual control input $u$. It can be seen from the figure, compared to removing variable-parameter predefined performance, the 


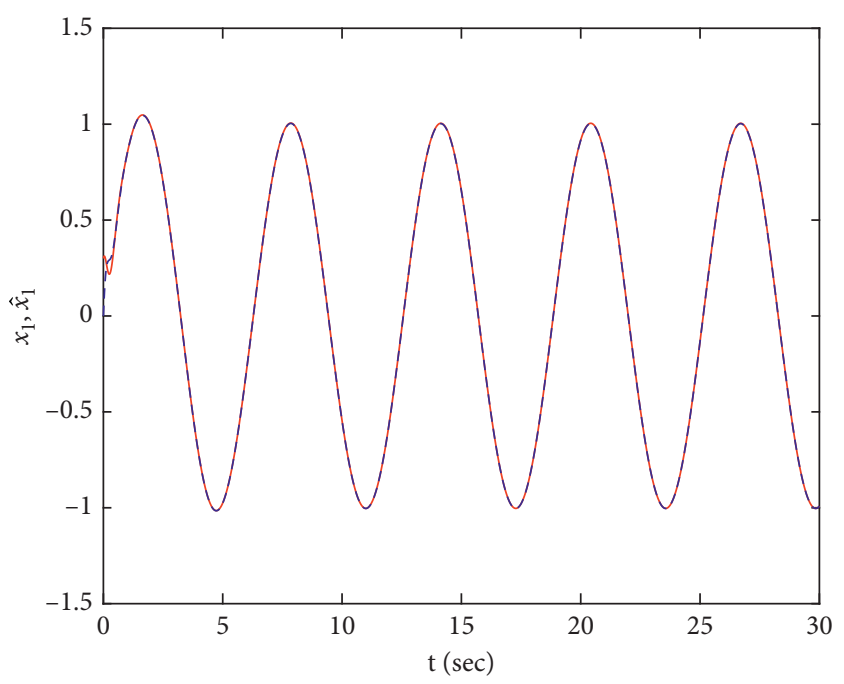

$\begin{array}{ll}- & x_{1} \\ --- & \hat{x}_{1}\end{array}$

FIgURE 3: Trajectory of $x_{1}$ and $\widehat{x}_{1}$.

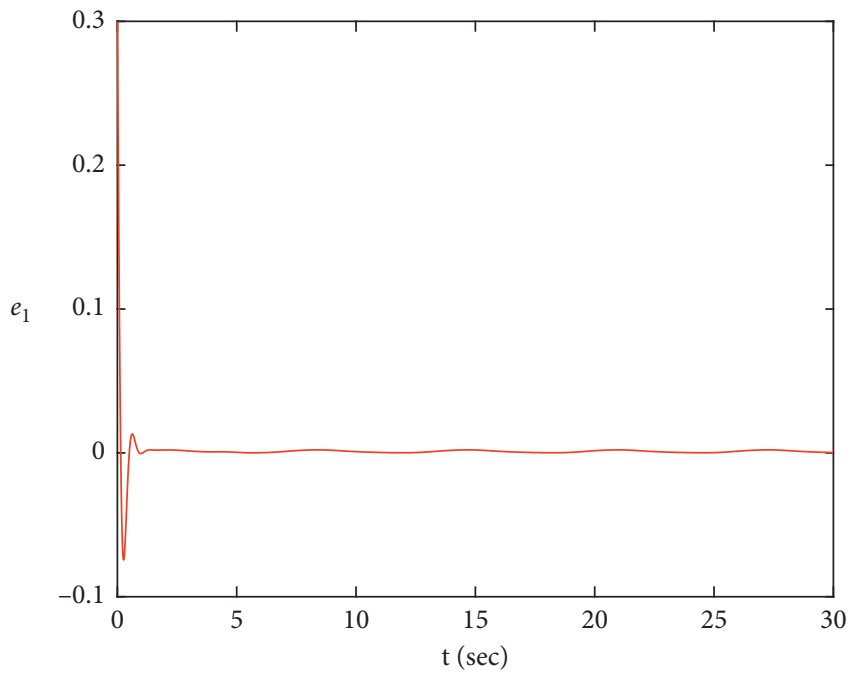

Figure 4: Tracking error $e_{1}$.

controller in this paper is obviously better in both the initial oscillation frequency and the tracking effect.

Remark 4. Compared with the existing literature, an adaptive fuzzy predefined performance controller is proposed in this paper, which makes the tracking error convergence in the preset range better. It can be seen from Figure 2 that different from the literatures [34,37], the novel

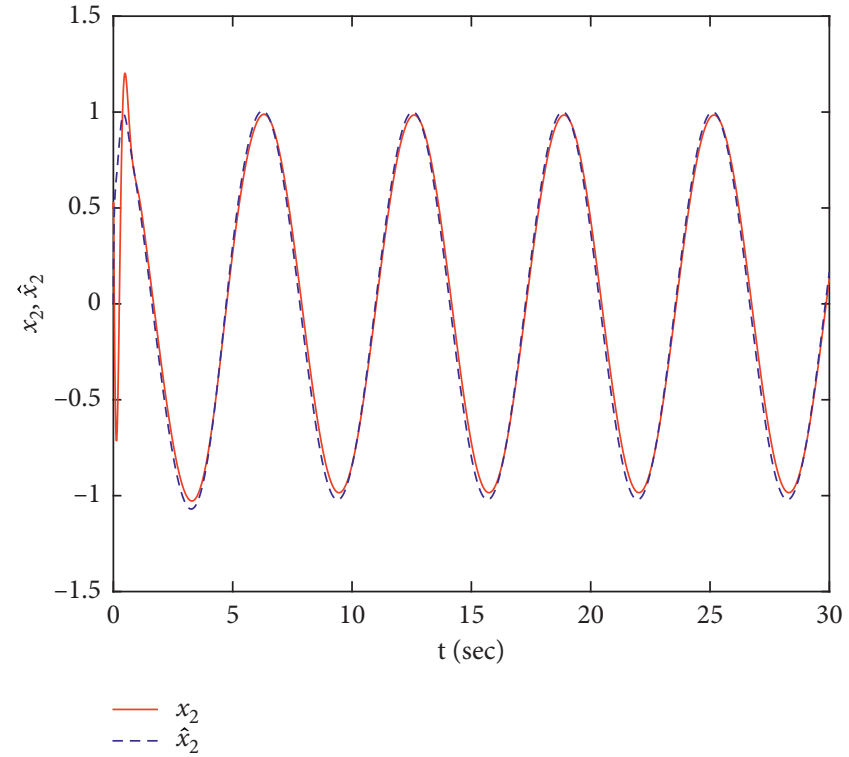

Figure 5: Trajectory of $x_{2}$ and $\hat{x}_{2}$.

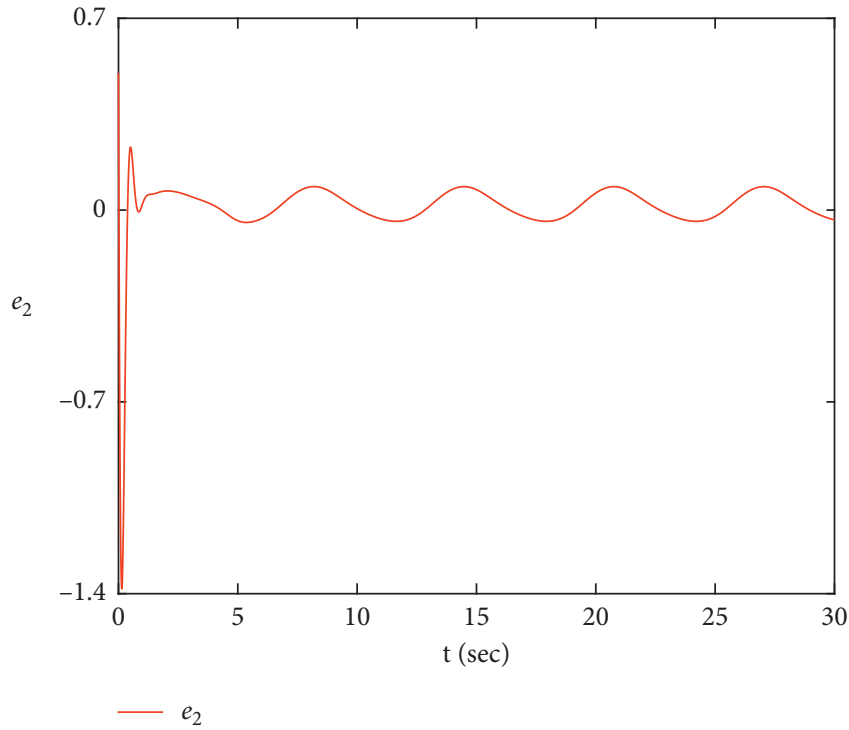

Figure 6: Tracking error $e_{2}$.

predefined performance control is a variable-parameter scheme, which relaxes the limitations of known initial error in predefined performance. In addition, from the simulation data, it can be seen that the simultaneous consideration of input delay and pure-feedback system brings great difficulty to controller design. The control method proposed in this paper has excellent control performance, which is shown in Figure 1. 


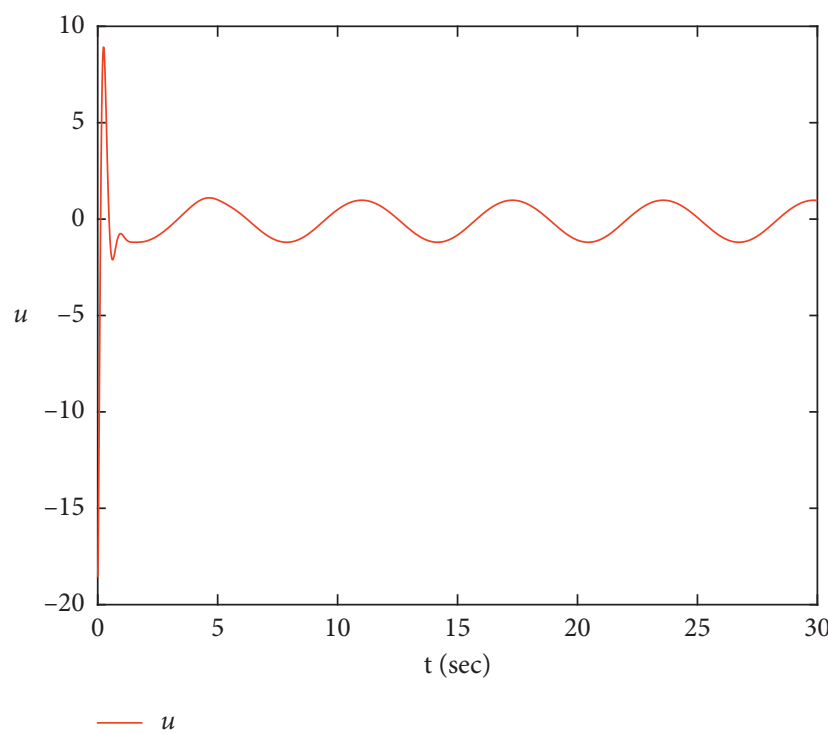

FIgURE 7: The trajectory of actual control input $u$.

\section{Conclusion}

In this paper, an observer-based adaptive fuzzy predefined performance controller has been introduced of a class of nonlinear pure-feedback systems with unknown control direction and input delay. A novel predefined performance with variable-parameter scheme has been investigated, which solved the problem of unknown initial error. In order to overcome system complexity caused by input delay and pure-feedback systems, the Pade approximation and mean value theorem has been employed, respectively. In addition, Nussbaum functions have been used to deal with the unknown control direction and a first-order filter has applied to approximate repeated differentiations problem of the virtual controllers. State observer and FLSs have been proposed to estimate the unmeasured states and approximate the unknown nonlinear functions, respectively. Therefore, it has been proved that stability of the entire closed-loop system is SGUUB in limitation of the predefined performance control. The tracking errors have converged within a predefined range, while the observer estimation errors have converged within a small zero region. Finally, the simulation results have verified the effectiveness of the proposed control method. In the future research, an observer-based adaptive fuzzy predefined performance controller will be considered in multiagent systems.

\section{Data Availability}

All data in this paper are from Simulink in MATLAB, and all data have been given in this paper.

\section{Conflicts of Interest}

The authors declare that they have no conflicts of interest.

\section{Acknowledgments}

This work was supported in part by the National Key R\&D Program of China (n. 2019YFE0105000), the National Natural Science Foundation of China under Grant (nos. 61773105, 61533007, 61873049, 61873053, 61703085, and 61374147), and the Fundamental Research Funds for the Central Universities of China under Grant no. N182008004.

\section{References}

[1] M. M. Polycarpou and P. A. Ioannou, "A robust adaptive nonlinear control design," Automatica, vol. 32, no. 3, pp. 423-427, 1996.

[2] B. Yao and M. Tomizuka, "Adaptive robust control of SISO nonlinear systems in a semi-strict feedback form," Automatica, vol. 33, no. 5, pp. 893-900, 1997.

[3] S.-I. Han and J.-M. Lee, "Recurrent fuzzy neural network backstepping control for the prescribed output tracking performance of nonlinear dynamic systems," ISA Transactions, vol. 53, no. 1, pp. 33-43, 2014.

[4] Z. Liu, F. Wang, Y. Zhang, and C. L. Philip Chen, "Fuzzy adaptive quantized control for a class of stochastic nonlinear uncertain systems," IEEE Transactions on Cybernetics, vol. 46, no. 2, pp. 524-534, 2016.

[5] X. Gao, "Adaptive neural control for hysteresis motor driving servo system with bouc-wen model," Complexity, vol. 2018, Article ID 9765861, 9 pages, 2018.

[6] G. Cui, J. Yu, and Q.-G. Wang, "Finite-time adaptive fuzzy control for MIMO nonlinear systems with input saturation via improved command-filtered backstepping," IEEE Transactions on Systems, Man, and Cybernetics: Systems, vol. 99, pp. 1-10, 2020.

[7] G. Cui, W. Yang, and J. Yu, "Neural network-based finite-time adaptive tracking control of nonstrict-feedback nonlinear systems with actuator failures," Information Sciences, vol. 545, pp. 298-311, 2021.

[8] D. Swaroop, J. Gerdes, P. Yip, and J. Hedrick, "Dynamic surface control of nonlinear systems," Proceedings of the 1997 American Control Conference (Cat. No.97CH36041), vol. 5, pp. 3028-3034, 1997.

[9] D. Wang and J. Huang, "Neural network-based adaptive dynamic surface control for a class of uncertain nonlinear systems in strict-feedback form," IEEE Transactions on Neural Networks, vol. 16, no. 1, pp. 195-202, 2005.

[10] L. Ma, N. Xu, X. Huo, and X. Zhao, "Adaptive finite-time output-feedback control design for switched pure-feedback nonlinear systems with average dwell time," Nonlinear Analysis Hybrid Systems, vol. 37, 2020.

[11] X. Fan, Y. Wang, L. Wu, and X. Zhang, "An r-order finitetime state observer for reaction-diffusion genetic regulatory networks with time-varying delays," Complexity (New York, N.Y.), vol. 2018, Article ID 7257083, 15 pages, 2018.

[12] Q. Shen, P. Shi, and Y. Shi, "Distributed adaptive fuzzy control for nonlinear multiagent systems via sliding mode observers," IEEE Transactions on Cybernetics, vol. 46, no. 12, pp. 30863097, 2016.

[13] J. Du, X. Hu, H. Liu, and C. L. P. Chen, "Adaptive robust output feedback control for a marine dynamic positioning system based on a high-gain observer," IEEE Transactions on Neural Networks and Learning Systems, vol. 26, no. 11, pp. 2775-2786, 2015. 
[14] Y. Chang, Y. Wang, F. E. Alsaadi, and G. Zong, "Adaptive fuzzy output-feedback tracking control for switched stochastic pure-feedback nonlinear systems," International Journal of Adaptive Control and Signal Processing, vol. 33, no. 10, p. 1567, 2019.

[15] Z.-M. Li and X.-H. Chang, "RobustHoocontrol for networked control systems with randomly occurring uncertainties: observer-based case," ISA Transactions, vol. 83, pp. 13-24, 2018.

[16] Q. Shen, B. Jiang, and V. Cocquempot, "Adaptive fuzzy observer-based active fault-tolerant dynamic surface control for a class of nonlinear systems with actuator faults," IEEE Transactions on Fuzzy Systems, vol. 22, no. 2, pp. 338-349, 2014.

[17] S. Wang, X. Ren, and J. Na, "Adaptive dynamic surface control based on fuzzy disturbance observer for drive system with elastic coupling," Journal of the Franklin Institute, vol. 353, no. 8, pp. 1899-1919, 2016.

[18] S. Tong, Y. Li, G. Feng, and T. Li, "Observer-based adaptive fuzzy backstepping dynamic surface control for a class of MIMO nonlinear systems," IEEE Transactions on Systems, Man, and Cybernetics, Part B (Cybernetics), vol. 41, no. 4, pp. 1124-1135, 2011.

[19] Z. Liu, X. Dong, J. Xue, H. Li, and Y. chen, "Adaptive neural control for a class of pure-feedback nonlinear systems via dynamic surface technique," IEEE Transactions on Neural Networks and Learning Systems, vol. 27, no. 9, pp. 1969-1975, 2016.

[20] C. Xi, D. Zhai, J. Dong, and Q. Zhang, “Approximation-based adaptive fuzzy tracking control for a class of switched nonlinear purefeedback systems," International Journal of Systems Science, vol. 354, no. 15, pp. 6567-6587, 2017.

[21] Y. Wen and X. Ren, "Observer-based fuzzy adaptive control for non-linear time-varying delay systems with unknown control direction," IET Control Theory \& Applications, vol. 4, no. 12, pp. 2757-2769, 2010.

[22] C.-C. Wang and G.-H. Yang, "Observer-based adaptive prescribed performance tracking control for nonlinear systems with unknown control direction and input saturation," Neurocomputing, vol. 284, pp. 17-26, 2018.

[23] H. Wu, S. Liu, C. Cheng, and C. Du, "Observer based adaptive double-layer fuzzy control for nonlinear systems with prescribed performance and unknown control direction," Fuzzy Sets and Systems, vol. 392, pp. 93-114, 2020.

[24] J. Ma, S. Xu, Q. Ma, and Z. Zhang, "Event-Triggered adaptive neural network control for nonstrict-feedback nonlinear time-delay systems with unknown control directions," IEEE Transactions on Neural Networks and Learning Systems, vol. 31, no. 10, pp. 4196-4205, 2020.

[25] T. Zhang and S. Ge, "Adaptive dynamic surface control of nonlinear systems with unknown dead zone in pure feedback form," Automatica, vol. 44, no. 7, pp. 1903-1985, 2008.

[26] W. Si and X. Dong, "Adaptive neural control for MIMO stochastic nonlinear pure-feedback systems with input saturation and full-state constraints," Neurocomputing, vol. 275, pp. 298-307, 2018.

[27] Y. Li, S. Tong, and T. Li, "Adaptive fuzzy output-feedback control for output constrained nonlinear systems in the presence of input saturation," Fuzzy Sets and Systems, vol. 248, pp. 138-155, 2014.

[28] J. Na, X. Ren, C. Shang, and Y. Guo, "Adaptive neural network predictive control for nonlinear pure feedback systems with input delay," Journal of Process Control, vol. 22, no. 1, pp. 194-206, 2012.
[29] D.-P. Li, Y.-J. Liu, S. Tong, C. L. P. Chen, and D.-J. Li, "Neural networks-based adaptive control for nonlinear state constrained systems with input delay," IEEE Transactions on Cybernetics, vol. 49, no. 4, pp. 1249-1258, 2019.

[30] Q. Zhang and J. Dong, "Disturbance-observer-based adaptive fuzzy control for nonlinear state constrained systems with input saturation and input delay," Fuzzy Sets and Systems, vol. 392, pp. 77-92, 2020.

[31] H. Li, L. Wang, H. Du, and A. Boulkroune, "Adaptive fuzzy backstepping tracking control for strict-feedback systems with input delay," IEEE Transactions on Fuzzy Systems, vol. 25, no. 3, pp. 642-652, 2017.

[32] L. Ma, X. Huo, X. Zhao, and G. Zong, “Adaptive fuzzy tracking control for a class of uncertain switched nonlinear systems with multiple constraints: a small-gain approach," International Journal of Fuzzy Systems, vol. 21, no. 9, pp. 2609-2624, 2019.

[33] J. Cai, R. Yu, B. Wang, C. Mei, and L. Shen, "Decentralized event-triggered control for interconnected systems with unknown disturbances," Journal of the Franklin Institute, vol. 357, no. 3, pp. 1494-1515, 2019.

[34] C. Cheng, Y. Zhang, and S. Liu, "Neural observer-based adaptive prescribed performance control for uncertain nonlinear systems with input saturation," Neurocomputing, vol. 370, pp. 94-103, 2019.

[35] J. Ni, C. K. Ahn, L. Liu, and C. Liu, "Prescribed performance fixed-time recurrent neural network control for uncertain nonlinear systems," Neurocomputing, vol. 363, no. 21, pp. 351-365, 2019.

[36] F. Jia, X. Yan, X. Wang, J. Lu, and Y. Li, "Robust adaptive prescribed performance dynamic surface control for uncertain nonlinear pure-feedback systems," Journal of the Franklin Institute, vol. 357, no. 5, pp. 2752-2772, 2020.

[37] Y. Liu, X. Liu, and Y. Jing, "Adaptive neural networks finitetime tracking control for non-strict feedback systems via prescribed performance," Information Sciences, vol. 468, pp. 29-46, 2018.

[38] C. Gao, C. Zhang, X. Liu, H. Wang, and L. Wang, "Eventtriggering based adaptive neural tracking control for a class of pure-feedback systems with finite-time prescribed performance," Neurocomputing, vol. 382, pp. 221-232, 2020.

[39] J. Luo, C. Wei, H. Dai, and J. Yuan, "Robust LS-SVM-based adaptive constrained control for a class of uncertain nonlinear systems with time-varying predefined performance," Communications in Nonlinear Science and Numerical Simulation, vol. 56, pp. 561-587, 2018.

[40] L. Wang, Adaptive Fuzzy Systems and Control, Prentice-Hall, Englewood Cliffs, NJ, USA, 1994.

[41] Z. Ramezani, M. M. Arefi, H. Zargarzadeh, and M. R. JahedMotlagh, "Neuro observer-based control of pure feedback MIMO systems with unknown control direction," IET Control Theory \& Applications, vol. 11, no. 2, pp. 213-224, 2017.

[42] W. Chen, X. Li, W. Ren, and C. Wen, "Adaptive consensus of multi-agent systems with unknown identical control directions based on A novel nussbaum-type function," IEEE Transactions on Automatic Control, vol. 59, no. 7, pp. 18871892, 2014. 